\title{
SYNCHRONIZATION OF KURAMOTO OSCILLATORS WITH DISTANCE-DEPENDENT DELAY*
}

\author{
Karol Trojanowski ${ }^{\dagger}$, Lech LOngA ${ }^{\ddagger}$ \\ The Marian Smoluchowski Institute of Physics \\ Division of Statistical Physics \\ and \\ The Mark Kac Complex Systems Research Center \\ Jagiellonian University, Reymonta 4, Kraków, Poland
}

(Received May 20, 2013)

We investigate the synchronization process in a Kuramoto model of phase-coupled oscillators with distance-dependent delay. The oscillators occupy the nodes of a two-dimensional square lattice subjected to periodic boundary conditions. The mean-field interactions with velocity-dependent delays propagate along the lattice sites. This gives rise to a non-uniform distribution of delays and lattice dimensionality dependence, which is not present in mean-field models without delays. We find that the 'coupling strength-delay' phase diagram does not show up reentrant behavior present in models with uniform delay. A number of dynamic patterns, reported earlier for a generalized Kuramoto model with non-mean-field distancedependent interactions, is also found.

DOI:10.5506/APhysPolB.44.991

PACS numbers: 05.45.Xt, 87.19.1j, 89.75.-k

\section{The model}

The popular Kuramoto model of mutual synchronization of coupled oscillators [1] has, since its inception, drastically improved the understanding of this prevalent phenomenon. Common examples [2,3] include synchronous chirping of crickets, flashing of Chinese fireflies [4], clapping of audiences, bursting of neurons, contraction of heart muscles or operation of Josephson

* Presented at the XXV Marian Smoluchowski Symposium on Statistical Physics, "Fluctuation Relations in Nonequilibrium Regime", Kraków, Poland, September 10-13, 2012.

† karol.trojanowski@uj.edu.pl

$\ddagger$ lech.longa@uj.edu.pl 
junction arrays $[5,6]$, to name a few. This model still remains the most successful one, due to its mathematical tractability, combined with the ability to capture the essence of synchrony.

We build up from the definition of the Kuramoto model, which is most suitable for direct treatment by numerical methods [2, 7]

$$
\dot{\theta}_{i}(t)=\omega_{i}+\frac{K}{N} \sum_{j=1}^{N} \sin \left(\theta_{j}(t)-\theta_{i}(t)\right),
$$

where $i=1 \ldots N, \theta_{i}(t)$ is the phase of the $i$ th oscillator at time $t$ and $\omega_{i}$ are intrinsic oscillator frequencies, sampled from yet unspecified probability distribution $\rho(\omega)$ on compact support. Kuramoto solved this model exactly in the case of $N \rightarrow \infty$ and $\omega_{i}$ sampled from a Lorentz distribution. Solutions for other distributions have subsequently been obtained. A model, so defined, exhibits a (mean-field-type) phase transition between the disordered (incoherent) and ordered (coherent) phases, as the coupling constant $K$ is increased. Order is monitored by the real parameter $r$, defined as

$$
r(t) e^{i \psi(t)}=\frac{1}{N} \sum_{j=1}^{N} e^{i \theta_{i}(t)} .
$$

When the stationary state is assumed, $r(t)=r(r \in[0,1])$, with $r=1$ and $r=0$ in total coherence and incoherence, respectively.

Some real systems cannot be considered without taking delay into account. The popular example of a clapping audience synchronizing to clap in unison is valid only for sufficiently small audiences, such as opera halls. When distances are of the order of $300 \mathrm{~m}$, or higher, the finite speed of sound makes the delay non-negligible. As a result, e.g. football arena audiences cannot clap together or have difficulty in coherent singing.

We start by introducing delay to (1) in the most general way

$$
\dot{\theta}_{i}(t)=\omega_{i}+\frac{K}{N} \sum_{j=1}^{N} \sin \left(\theta_{j}\left(t-\tau_{i j}\right)-\theta_{i}(t)\right) .
$$

The case of uniform delay, $\tau_{i j} \equiv \tau$, is interpreted as coupling of the state at $t$ to the state at $t-\tau$. The stability of incoherence in such a model has been studied by Yeung and Strogatz in [8] and by others [9-11].

However, in most situations, the delays are not all identical. The more realistic case, with distributed time delays [12, 13], shows that spread in the distribution function of delays can greatly alter the system dynamics. 
We introduce non-uniform delay by arranging the nodes on a square lattice while preserving the topology of a complete graph. The coupling remains uniform, however the interaction propagates along the lattice sites with the delay being distance-dependent through the definition

$$
\tau_{i j}=\tau \frac{s_{i j}}{\left\langle s_{i j}\right\rangle}
$$

where $\tau$ is interpreted as the inverse velocity. The distance $s_{i j}$ is defined with the so-called "taxi-driver's measure", i.e. as sum of the differences in horizontal and vertical coordinates and is measured in number of nodes. To maintain translational invariance we identify the opposite edges and the shortest route is always preferred. Hence, when $N=L \times L$, the average distance between any pair of nodes $\left\langle s_{i j}\right\rangle=\frac{L}{2}$. This definition normalizes the maximum delay to $2 \tau$ and removes dependence on the network size. We find that the unmodified parameter $r$, defined by (2), is useful in monitoring the average order in the sample.

\section{Simulations and results}

We have investigated the behavior of the model described by (3) and (4) by integrating the equations (3) using a four-step Adams-Bashforth scheme. For simplicity and reference with previous results [8], we set $\omega_{i}=\frac{\pi}{2}$ for all $i$, therefore $\rho(\omega)=\delta\left(\omega-\frac{\pi}{2}\right)$. A run for one pair of parameters $(\tau, K)$ consisted of 10000 integration steps with step size $\Delta t=0.01$, out of which the last 6000 were considered for averaging the order parameter $r$ to obtain the temporal average $\bar{r}$, rejecting the first 4000 when the system is approaching stability. Lattices as large as $32 \times 32$ were considered. The initial conditions, as well as histories of $\theta_{i}$, were sampled uniformly from $[0,2 \pi)$.

We have found phase boundaries between the completely ordered and disordered phase (Fig. 1 (right)). For reference, we have produced a diagram for $\tau_{i j} \equiv \tau$ (Fig. 1 (left)). In the case of $\rho(\omega)=\delta\left(\omega-\omega_{0}\right)$, the transitions occurring with changing $\tau$ are instantaneous. It is observed that non-uniform delay removes the reentrance of synchrony, as intuitively expected. This difference is easily understood when considering low coupling. The reentrance in the case of uniform delay and $K \ll 1$ is due to there being sufficiently little difference between states at $t-\tau$ and $t$ for the effect of delay to be approximated by the rotation of all oscillators with average frequency

$$
\sin \left(\theta_{j}(t-\tau)-\theta_{i}(t)\right) \approx \sin \left(\theta_{j}(t)-\theta_{i}(t)-\bar{\omega} \tau\right) .
$$

The reentrance for low coupling then occurs when $\bar{\omega} \tau$ is close to an integer multiple of $2 \pi\left(\tau \approx k \frac{2 \pi}{\bar{\omega}}, k=1,2, \ldots\right)$, where the low-delay limit is reproduced. Our simulations reflect this heuristic quantitatively up to $k=2$ 

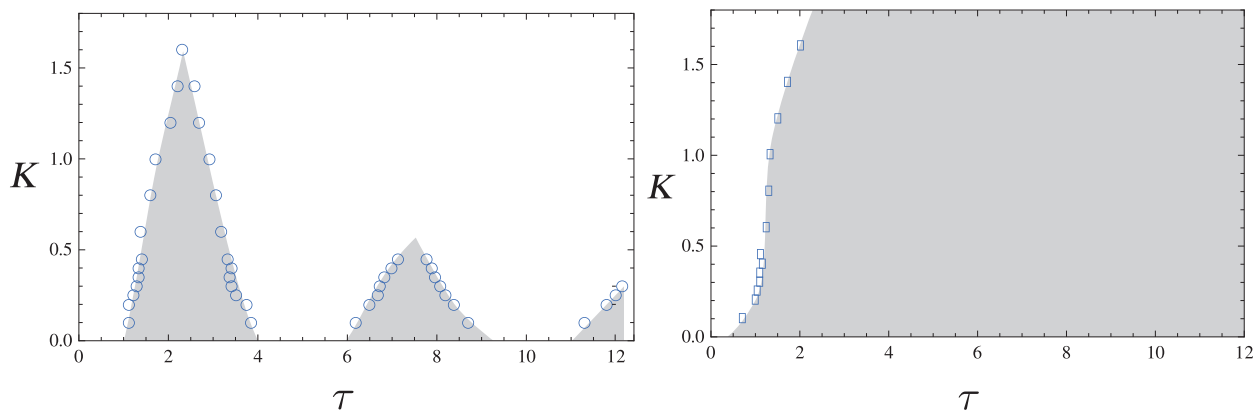

Fig. 1. Phase diagram portions for the uniform delay model (left) and the model described by (4) (right). The shaded areas visually approximate the incoherent regimes. The phase border points are results of numerical integration of (3).

and qualitatively from $k=3$ on. In the case of distance-dependent delay (Fig. 1 (right)) and $K \ll 1$, the effects of delay are individually approximated by phase shifts of $\bar{\omega} \tau \frac{s_{i j}}{\left\langle s_{i j}\right\rangle}$ which vary across connections and the low-delay limit cannot be reproduced by a specific value of delay. Hence, no reentrance occurs. Interestingly, in the synchronized regime, we observe a formation of dynamical patterns (traveling phase rolls and phase squares). Exemplary snapshots are shown in Fig. 2. The full outcome of the model, including analytical results, will be presented elsewhere.
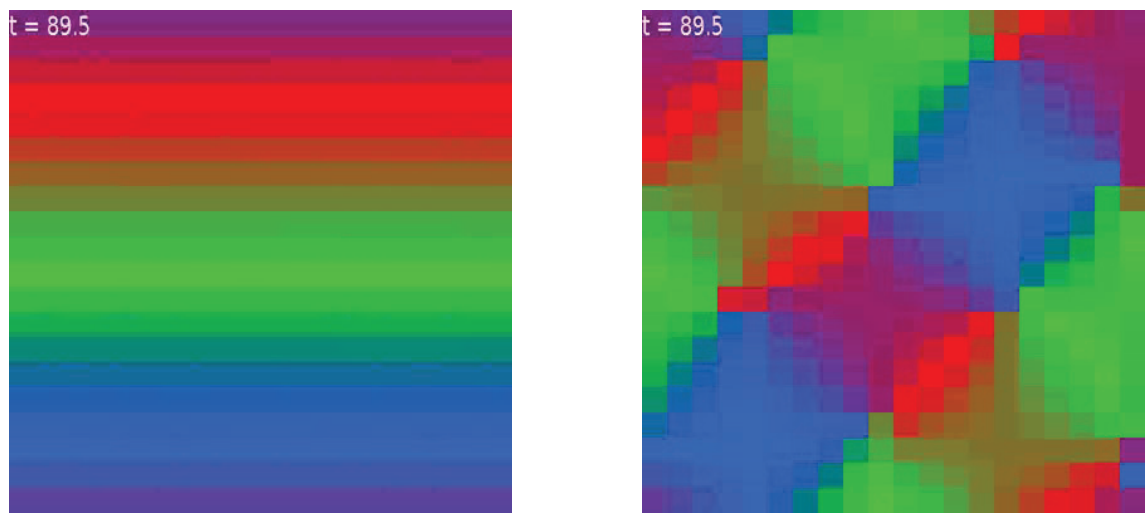

Fig. 2. (Color online) Dynamical patters formed by phases in the Kuramoto model for $K=0.8$ : (left) $\tau=1.1$ and (right) $\tau=1.9$. The snapshots were obtained for $N=20 \times 20$ lattice with the initial phases set at random from a uniform distribution on $[0,2 \pi)$. Shown are the steady states viewed from a reference frame rotating with the average frequency of the sample $\Omega=1 / N \sum_{i} \dot{\theta}_{i}$. The phase is color-coded, with black/red for 0 and $2 \pi$, light grey/green for $\pi / 3$ and grey/blue for $2 \pi / 3$. Note that the characteristic size of the structure or wavelength decreases with increasing $\tau$. 
The project operated within the Foundation for Polish Science International Ph.D. Projects Programme co-financed by the European Regional Development Fund covering, under the agreement No. MPD/2009/6, the Jagiellonian University International Ph.D. Studies in Physics of Complex Systems.

\section{REFERENCES}

[1] Y. Kuramoto, Chemical Oscillations, Waves and Turbulence, Springer Verlag, New York 1984.

[2] J.A. Acebron et al., Rev. Mod. Phys. 77, 137 (2005).

[3] S.H. Strogatz, Physica D 143, 1 (2000).

[4] J. Buck, E. Buck, Sci. Am. 234, 74 (1976).

[5] N.F. Pedersen, O.H. Soerensen, B. Dueholm, J. Mygind, J. Low Temp. Phys. 38, 1 (1980).

[6] K.A. Wiesenfeld, P. Colet, S.H. Strogatz, Phys. Rev. Lett. 76, 404 (1996).

[7] B.C. Daniels, published online, 2005, http://go.owu.edu/ physics/ StudentResearch/2005/BryanDaniels/kuramoto_paper.pdf

[8] M.K.S. Yeung, S.H. Strogatz, Phys. Rev. Lett. 82, 648 (1999).

[9] D.P.E. Montbrio, J. Schmidt, Phys. Rev. E74, 056201 (2006).

[10] K.G. Petrosyan, M.K. Sen, B.C. Bag, C.-K. Hu, J. Stat. Mech. 08, P08018 (2010).

[11] D.V. Senthilkumar, M.M. Shrii, J. Kurths, Eur. Phys. Lett. 98, 10003 (2012).

[12] E. Ott, W.S. Lee, T.M. Antonsen, Phys. Rev. Lett. 103, 044101 (2009).

[13] U. Munza, G.S. Schmidt, A. Papachristodoulou, F. Allgower, Automatica 48, 3008 (2012). 